link in the chain whereby muscular action leads to increased secretory activity and increased tensicn.

3. Muscular action is the cause, where a staphyloma has been developed, of variations in its volume, and likewise of the alternation of traction and relaxation to which the iris is subject. Reasoning from what we know of the different effects of interrupted and continuous irritation, it is to be inferred that a varying traction is much more likely to cause increased secretion than a constant unvarying traction.

4. Muscular action explains the loss of vision which occurs in distended eyes with prominent cicatrix but without any adhesion of the iris.

It remains now to consider the treatment from the stage of perforation to the full development of the staphyloma. In case of perforation of the cornea with prolapse of the iris, were it possible to paralyse all the ocular muscles until the cicatrisation should be completed, doubtless staphyloma would be much more rare. Compulsory rest, secured by a compress bandage, is the nearest approach to this, and affords at the same time support to the weak cornea, and favours the formation of a firm cicatrix. It is not enough, however, to rest satisfied with the application of a bandage. The patient must be directed to move the uncovered eye as little as possible, because the muscles of the two ejes act in harmony, and even although those of the bandaged eye may not be able to cause movement, yet they will exert pressure, and a pressure great in proportion to the resistance offered by the bandage to motion. The pressure of the bandage when properly applied, however, supports the weak part so that it will not bulge from the muscular action. If, however, the bandage should happen to be relaxed, then the eye may move and the bulging may take place. The importance of rest of the uncovered eje will be better understood when it is borne in mind how difficult it is to secure the firm application of a bandage for many hours at a time, and how often during the day and night it may require readjustment.

With hospital patients it is necessary that the instructions be very precise and peremptory, and the evil results of any departure must be forcibly represented. The necessity for this caution is shown by the following case. A lad, ten years of age, was under my care for ulcers at the lower part of the cornea of both eyes. The case progressed unfavourably without any ascertained cause, and terminated in perforation, prolapse, and staphyloma. I casually learned that the compress bandage which I applied daily was removed frequently during the day to allow the friends and acquaintances an opportunity of examining the eyes. Thus ignorance interfered to render the treatment useless. Fortunately, however, by excision of a small portion of the staphylomatous tissue and application of compress the boy recovered with little damage to vision and total subsidence of the staphyloma. The period during which the compress should be applied must be regulated by the circumstances of each case. The larger the perforation the longer will compression be necessary. The time should vary from one to two or three months. This will not appear unnecessarily long when the period during which consolidation and retraction of cicatricial tissue proceed is borne in mind. It is desirable by direct means to diminish the irritability of the projecting part and lessen its size, so that the compress may exert its influence under more favourable circumstances. The section of the base of the staphyloma at its sclerotic margin has more permanent effect than a simple tapping, and has the great advantage of severing the nerves of the iris and cornea involved in the staphyloma, and is therefore always to be preferred. Excision of a small portion of the tissue also favours firm cicatrisation, and is useful by removing a redundancy of tissue, the surface of the prolapsed iris being always greater than the part of the cornea destroyed.

Suppose, however, that, notwithstanding all our efforts, the cicatrix which results has not sufficient resisting power to withstand the internal pressure during muscular action; that the cicatrix becomes prominent; and that, through its dragging upon the iris, increased secretory activity is set up, and a condition of secondary glaucoma impending or established, - then iridectomy must be performed. The mode of its performance is a matter of the first importance. One writer recommends that the iridectomy be made at the part directly opposite to the staphyloma, with a view of diverting part of the pressure from the staphyloma towards the centre of the cornea and a part to the cornea in front of the excised iris. I confess I fail to see in what way such a decomposition of the intraocular pressure can be expected, the muscular force and the relative resisting power of the different parts of the cornea remaining unchanged after the operation. No doubt it is leneficial, but for a very different reason. The part of the iris furthest from the staphyloma is often more stretched than any other, and therefore from its excision relief may be expected. Besides, tension is relieved. Most writers direct an iridectomy without defining where. I propose a definite plan to be followed in all cases-namely, the excision of the portion of the iris adjacent to the staphyloma, as in the second and third operations in the first case reported. In bad cases an iridectomy should be performed on each side of the staphyloma, allowing an interval of a week to elapse between the two operations. When the staphyloma is small and the traction exerted upon only a portion of the iris, excision of the stretched portion will suffice, as in Case 2. For this plan I claim the advantage of securing the healthy iris, which may be allowed to remain in the eye, from the possibility of any traction during temporary distension of the staphyloma, whether from muscular action or any other cause, and at the same time of diminishing the tension as effectually as if performed at any other part.

I am well aware that the performance of the operation in this way is attended with some difficulty, because of the close approximation of the cornea and iris adjacent to the staphyloma. Unless union between these structures should have actually occurred, by means of Graefe's narrow cataract knife and scissors the necessary section can be made. It is urgent to perform the operation as early as possible. Although this leaves muscular action untouched, jet it breaks the chain of its morbid influence.

If the disease should still progress, I would make a section of either the internal or external rectus, or both. I have not yet had occasion to resort to this, but will try it in the first case requiring such a measure.

Use of the eyes in reading, sewing, and regarding minute objects should be forbidden for a period varying from three months to one year.

In case any confusion should be experienced from difference in the size and shape of the retinal images of the two eyes, the patient should be relieved either by a suitable glass if the difference be slight in degree, or by an opaque glass opposite the damaged eye, to exclude it altogether from vision for a time.

Belfast.

\section{ENTERIC FEVER LATENS.}

\section{By WILLTAM ROBERT WALL, F.R.C.S.I., SURGEON, ABMY MEDICAL DEPARTMENT.}

ENTERIC FEVER of a masked or latent type is becoming a recognised fact, and has been described by Bristowe, Murchison, and other writers,-all the usual symptoms (such as rose-coloured eruption, pain and gurgling in the right iliac fossa, meteorism, diarrhœa, and intestinal hæmorrhage) being absent until fatal perforation of the intestine takes place. This was remarkably illustrated by a case which occurred at Neemuch, Rajpootana.

A healthy young woman, aged twenty-four, the wife of a sergeant, was suddenly seized with intense pain in the abdomen. She stated that she had just returned from an entertainment, and that she had felt quite well for the last three weeks, and able to look after her family. The bowels were more or less constipated during that period. Symptoms of peritonitis immediately supervened, with profound collapse; and she died sixteen hours after being attacked.

Post-mortem appearances.-Peritoneum intensely inflamed. The anatomical sign of typhoid fever was well marked, the lower third of the ileum containing several ulcers, elliptical and circular in shape, one of which had completely perforated the intestine, allowing the contents of the gut to escape into the peritoneal cavity. The mucous membrane was in a state of catarrhal inflammation.

Previous to this woman's death there were two wellmarked cases of enteric fever in the same regiment. I have 
treated several cases of this insidious malady as " continued and remittent fever" until a few days before the fatal termination, when diarrhoea and collapse set in rapidly, causing death, the post-mortem showing perforating ulcer in the lower part of the ileum. Several cases of this disease have occurred at this station (Mhow) lately.

J. J-, admitted April 7th, suffering from what appeared to be remittent fever. Pulse weak and frequent, slight diarrboa, no gurgling, or "rose-coloured" spots. Twelve days after admission he suddenly got much worse; pulse scarcely perceptible, sordes on teeth, no diarrhoea or eruption. Died twelve days after admission.

Sectio cadaveris.-The whole of the ileum was in a very congested state, Peyer's patches very much thickened, and towards the ileo-cacal valve, ulcerated.

A case of well-marked typhoid fever was admitted on the 18th April. Rose-coloured spots, severe diarrbcea, abdomen tympanitic, tongue very dry and brown. Died thirty days after admission. The post-mortem discovered almost the same lesions as in the former case.

I. T-, admitted on June 1st for remittent fever. Bowels regular; skin hot; generally one or two degrees $F$. increase in evening temperature; no eruption; pulse quick and small. Low muttering delirium set in, and he died twenty-eight days after admission.

Post-mortem.-The lower part of ileum covered with large patches of ulceration, and in one or two places nearly ulcerated.

In this case there was no eruption or diarrbœa. Temperature three days before fatal termination:-June 25th: morning, $96^{\circ}$ F.; evening, $98^{\circ}$. 26th: morning, 97 evening, $98^{\circ}$. 27th : morning, $98^{\circ}$; evening, $992^{\circ}$.

Until comparatively of late years the existence of typhoid fever has been denied in India, and I have no doubt that cases of that type have been returned as "continued" and remittent fever. A question of vital importance now arises: What are the causes of this disease, especially in India? As far as I can see (in addition to the defective removal of excreta, \&c.) the wells in this country are quite uncovered, and only surrounded by a low wall, on which it is no uncommon sight to see the "water-carriers" washing their clothes and performing ablutions. During dust-storms, and the strong winds that prevail (generally S.W. in Bombay presidency), a variety of foreign substances, such as organic matter, "cholera and enteric dust," and low organisms, must necessarily find their way into the drinkingwater used by the soldiers, where they have all the essential conditions for decomposition - i. e., moisture, access of atmospheric air, and the rays of a tropical sun. A simple and inexpensive remedy for this defect might be found by placing a raised roof of thatch over each well, and also an enclosure of the same to the windward side (the entrance being to leeward). This would allow the free access of air, and also protect the water from impurities in the atmosphere.

This form of enteric fever being so obscure, and liable to be mistaken for remittent fever, makes it difficult to diagnose. I think the most certain sign is the evening increase of temperature as shown by the clinical thermometer, often very slight (not more than one or two degrees above $98^{\circ} \mathrm{Fahr}$.) In places where enteric fever is endemic the morning and evening temperature in all cases of fever should be carefully recorded, and even a slight increase in the evening temperature looked on as suspicious. As regards the treatment, $I$ think the advice given by Graves ("anticipate symptoms") in the treatment of fevers generally peculiarly suited to this form of disease ; and although there may be no rose-coloured spots, pain, tenderness, or gurgling in the right iliac fossa, commence at once by topical depletion, application of ice-bladders, \&c., in this region; keep the patient strictly in the recumbent position; and be most particular as regards diet; no solids being allowed as long as the temperature of the patient is above the normal standard.

Mhow.

Catarkh of the Fallopian Tubes. - In the American Journal of Obstetrics Dr. Hennig expresses the opinion that catarrh of the Fallopian tubes is more frequent than that of any other part of the female sexual organs. Out of 103 females he found catarrh of the tubes present in 44. It occurs oftener before than after the critical period in married women.

\section{CLINICAL OBSERVATIONS ON CONDITIONS} OF THE FEMALE BLADDER;

SHOWING THE ADVANTAGES TO BE GAINED BY THE

USE OF A SELF-RETAINING FEMALE CATHETER.

By THOMAS STRETCH DOWSE, M.D., F.R.C.P., MBDICAL SURERTNTENDENT, CENTRAL LONDON SICK ASYLUM, HIGHGATE.

UNTIL about three years ago, when Mr. Barnard Holt first brought before the notice of the profession an elastic winged self-retaining male catbeter, notbing had been done to secure that which was so greatly needed-the self-retention of the catheter in the bladder. It seems strange that so long a period of scientific progress in the art of medicine and surgery should have elapsed before something of this kind (simple though it be) was brought into use.

Shortly after I became attached to this institution, where I have one ward of thirty-two beds occupied almost exclusively by palsied female patients, the necessity of some means to collect the urine which involuntarily made its escape from the paralysed bladder at once became evident. And, after having tried with little success a variety of urinals, sponges, and bags, the thought occurred to me that a modification of the male self-retaining catheter adapted to the female bladder would be of incalculable advantage to both physician and patient. The bladder being the receptacle for one of the most varying fluids secreted in the animal economy - a fluid, too, which is more prone to rapid decomposition than any other, especially when brought into contact with animal tissues in a state of metamorphosis, the urine is not only by this means changed itself, but by a process of catalysis reacts upon the healthy portion of the bladder tissue, producing a chronic condition of disease which almost inevitably leads to serious, if not to fatal, results.

In acute cystitis the great pain arises not so much from the inflamed condition of the walls of the bladder as it does from the reflex muscular movements produced by the irritable condition and inco-ordinate power of the muscular sphincter. In the height of the inflammatory stage of acute cystitis it would scarcely be found advantageous to use a self-retaining catheter, but as the disease becomes more chronic, and the walls of the bladder lose to a great extent, as they invariably do, their muscular power, the retention of a catheter will not only permit the complete escape of urine, but will at the same time keep the walls of the bladder passive; and to ensure that not only may ulcerated surfaces become healed, but the muscular tissue of the bladder itself will by being kept at perfect rest the more readily regain its normal function, and be less likely to lead to degenerative change.

In cystitis there are two conditions requiring special attention-first, the constant desire on the part of the irritable bladder to eject its contents, and, secondly, the inability of the sphincter to perfect its retention. Hence arises the incontinence of urine so general in these cases. Chronic cystitis supervenes in nine cases out of ten from a previous inflammatory attack, and from the pathological cause that all acute inflammations terminate either by resolution or decay, let that be molecular as in suppuration, or general as in sloughing. And it is at that period of the inflammatory attack, when the epithelial cells and mucous tissue are in a state wavering upon decay or repletion, that it is so necessary to prevent the slightest accumulation of urine in the bladder, which, if allowed, would inevitably lead to the most serious results. The following case is to the point.

M. S-, forty-three years of age, was admitted under my care about a year ago with hæmorrhage from the bladder accompanied with all the symptoms of acute inflammation, which continued for more than three weeks. As the general sympathetic pains in the loins, rectum, and hypogastric region abated, the urine became altered in character, was ropy, lost its hæmatoid condition, and soon became loaded with pus. There was some incontinence and incessant desire to micturate, accompanied always with great pain. Not agreeing with the means usually adopted in such cases, 